\title{
Enhanced off-center stellar tidal disruptions by supermassive black holes in merging galaxies
}

\author{
F.K. Liu ${ }^{1}$ and Xian Chen ${ }^{2,3}$
}

\begin{abstract}
Off-center stellar tidal disruption flares have been suggested to be a powerful probe of recoiling supermassive black holes (SMBHs) out of galactic centers due to anisotropic gravitational wave radiations. However, off-center tidal flares can also be produced by SMBHs in merging galaxies. In this paper, we computed the tidal flare rates by dual SMBHs in two merging galaxies before the SMBHs become self-gravitationally bounded. We employ an analytical model to calculate the tidal loss-cone feeding rates for both SMBHs, taking into account two-body relaxation of stars, tidal perturbations by the companion galaxy, and chaotic stellar orbits in triaxial gravitational potential. We show that for typical SMBHs with masses $10^{7} M_{\odot}$, the loss-cone feeding rates are enhanced by mergers up to $\Gamma \sim 10^{-2} \mathrm{yr}^{-1}$, about two order of magnitude higher than those by single SMBHs in isolated galaxies and about four orders of magnitude higher than those by recoiling SMBHs. The enhancements are mainly due to tidal perturbations by the companion galaxy. We suggest that off-center tidal flares are overwhelmed by those from merging galaxies, making the identification of recoiling SMBHs challenging. Based on the calculated rates, we estimate the relative contributions of tidal flare events by single, binary, and dual SMBH systems during cosmic time. Our calculations show that the off-center tidal disruption flares by un-bound SMBHs in merging galaxies contribute a fraction comparable to that by single SMBHs in isolated galaxies. We conclude that off-center tidal disruptions are powerful tracers of the merging history of galaxies and SMBHs.
\end{abstract}

Subject headings: black hole physics - galaxies: active - galaxies: kinematics and dynamics - galaxies: nuclei - gravitational waves

\section{Introduction}

In the $\Lambda$-cold dark matter cosmology, both dark matter halos and galaxies form due to frequent mergers. In this paradigm, hierarchical galaxy mergers would incorporate multiple supermassive black holes (SMBHs) into a galaxy (Volonteri et al. 2003). When two SMBHs, initially embedded in the two cores of the merging galaxies, sink to the common center of the system due to dynamical friction and become gravita-

\footnotetext{
${ }^{1}$ Department of Astronomy, Peking University, 100871 Beijing, China; fkliu@pku.edu.cn

${ }^{2}$ Kavli Institute for Astronomy and Astrophysics, Peking University, 100871 Beijing, China; chenxian@pku.edu.cn

${ }^{3}$ Max-Planck Institute for Gravitational Physics (Albert Einstein Institute), 14476 Golm, Germany
}

tionally bound, a supermassive black hole binary (SMBHB) would form (Begelman et al. 1980). During the interaction between the SMBHB and the stellar and gaseous environments, if the two SMBHs could successfully evolve to a separation of hundreds of Schwarzschild radius, then gravitational wave $(\mathrm{GW})$ radiation could lead to the coalescence of the SMBHs within a Hubble time, and the asymmetry of GW radiation is predicted to impart a recoiling velocity on the post-merger $\mathrm{SMBH}$ (Hughes 2009; Centrella et al. 2010). Detection of the GW radiation from coalescing SMBHB would be a vital test of the theory of general relativity (GR), and is the major goal of the ongoing Pulsar Timing Array (PTA) project and any future space-based GW mission.

Despite many efforts to detect GW radia- 
tion from coalescing SMBHBs, theoretical studies found large uncertainties for the dynamical evolution of SMBHB in normal galaxies: in the absence of gas and efficient stellar relaxation, the evolution of SMBHB would stall at sub-parsec (pc) scale and not enter the GW radiation regime (Merritt \& Milosavliević 2008; Colpi \& Dotti 2011), while recent $N$-body simulations suggest that efficient repopulation of stars to the galaxy core may be norm in real mergers (Preto et al. 2011; Khan et al. 2011). Observationally, it is difficult to test the dynamical evolution of SMBHB in stellar systems, because of the lack of electromagnetic (EM) radiation from the vicinity of the dormant SMBHs. Recently, "tidal flares", the EM outbursts produced due to tidal disruption of stellar objects by SMBHs, have been identified as powerful probes of the mass and spin of the otherwise dormant SMBHs (Rees 1988; Komossa 2002; Donlev et al. 2002; van Velzen et al. 2011; Gezari et al. 2009; Bloom et al. 2011; Burrows et al. 2011; Cenko et al. 2012). The flaring rate for a single SMBH in an isolated galaxy is estimated to be $10^{-5}$ to $10^{-4} \mathrm{yr}^{-1}$ (Magorrian \& Tremaine 1999; Sver \& Ulmer 1999; Wang \& Merritt 2004; Brockamp et al. 2011).

It is predicted that the formation and evolution of bound SMBHB at galaxy center would significantly change the event rate and affect the light curves of tidal flares. Shortly after the formation of SMBHB, the three-body interaction between the binary and a bound stellar cusp will enhance the flaring rate to as high as $1 \mathrm{yr}^{-1}$ (Ivanov et al. 2005; Chen et al. 2009, 2011; Wegg \& Nate Bode 2011). After the stellar cusp is disrupted, mainly due to slingshot ejection, if stellar relaxation is inefficient in galaxy center, the flaring rate will become one order of magnitude lower than that in the single black hole system (Chen et al. 2008). When the SMBHB enters the GW-radiation regime, the interruption and recurrence of tidal-flare light curve by the perturbing secondary black hole occur on an observable timescale (Liu et al 2009), and the stars resonantly trapped by the inspiralling SMBHB may produce a tidal flare around the coalescence of the binary (Schnittman 2010; Seto \& Muto 2011). After the coalescence of the binary, the launch of a recoiling SMBH might be also accompanied by a brief burst of tidal flares. As the recoil- ing SMBH travels outside the galaxy core, tidal disruption of the stars gravitationally bound to the hole may produce a flare apparently displaced from the galaxy center (Komossa \& Merritt 2008; Merritt et al. 2009; Stone \& Loeb 2012; Li et al. 2012). Because of the many differences between the flaring rates in single and binary SMBH systems, it is suggested that tidal flares can be utilized to constrain the fraction and dynamical evolution of SMBHBs in galaxy centers (Chen et al. 2008).

Off-nuclear tidal disruption flares and ultra compact star clusters with peculiar properties are suggested to be the key features of gravitational recoiling SMBHs in galaxies. However, off-center tidal disruption flares can also be produced by SMBHs in merging galaxies, and star clusters with the proposed peculiar characters may also form by tidal truncation of secondary galaxies in minor mergers. In particular, tidal flaring rates would be enhanced at a stage when the SMBHs are still isolated in the cores of merging galaxies because of the mutual perturbation between merging galaxies, much earlier than the formation of SMBHBs. Roos (1981) pioneered the discussions on the stellar tidal disruptions by assuming that merging galaxies harbor Sgr A*-like SMBHs and by taking into account perturbations by companion galaxies. However, it is unclear by how much the tidal disruption rates can be boosted in physical galaxy models combining the correlations of central SMBHs and galactic bulges, how many tidal flares in the universe are contributed by this merger phase, and how they would affect the constraint on the merger history of SMBHs. As a first step toward addressing these issues, in this paper we calculate the stellar-disruption rates during galaxy mergers and investigate the prospect of using tidal flares to probe multiple SMBHs in merging galaxies.

The outline of the paper is as follows. In $\S 2$ we introduce the basic loss-cone theory and the stellar-disruption process in single SMBH systems. In $\S 3$, we describe the stellar relaxation process in merging systems and generalize the losscone theory to calculate the corresponding stellardisruption rate. We also discuss our results for different merger parameters. Based on the calculated rates, we investigate the contribution of tidal fares by merging galaxies in $\S$ 国 and discuss 
our results and their implications in $\S 5$

\section{Loss-cone feeding in single SMBH sys- tem}

We first calculate stellar-disruption rates for isolated galaxies with single SMBHs, to prepare the basics for more complicated calculations for merging galaxies. A star with mass $m_{*}$ and radius $r_{*}$ would be tidally disrupted when it passes by an $\mathrm{SMBH}$ as close as the tidal radius

$$
\begin{aligned}
r_{t} \simeq & r_{*}\left(\frac{M_{\bullet}}{m_{*}}\right)^{1 / 3} \\
\simeq & 4.9 \times 10^{-6} M_{7}^{1 / 3} \times \\
& \left(\frac{r_{*}}{R_{\odot}}\right)\left(\frac{m_{*}}{M_{\odot}}\right)^{-1 / 3} \mathrm{pc}
\end{aligned}
$$

(Hills 1975; Rees 1988), where $M_{\bullet}$ is the black hole mass, $M_{7}=M_{\bullet} / 10^{7} M_{\odot}$, and $R_{\odot}$ and $M_{\odot}$ are, respectively, the solar radius and mass. In the following, we assume $r_{*}=R_{\odot}$ and $m_{*}=M_{\odot}$ unless mentioned otherwise. For these solar-type stars, when $M_{\bullet} \ll 4 \times 10^{7} M_{\odot}$, tidal disruption happens outside the marginally bound orbit of the black hole, and collisions between the bound stellar debris, as well as the subsequent accretion on to the black hole, could produce an EM flare known as the "tidal flare" (Rees 1988). The criterion for stellar disruption is then $J \leq$ $J_{\mathrm{td}} \simeq\left(2 G M_{\bullet} r_{t}\right)^{1 / 2}$, where $G$ is Newtonian gravitational constant, $J$ is the specific angular momentum, and $J_{\mathrm{td}}$ is the specific angular momentum corresponding to a pericenter distance of $r_{t}$. Here, the latter approximation accounts for the fact that most stars are disrupted along parabolic orbits, i.e., their specific binding energy $\mathcal{E} \ll$ $G M_{\bullet} / r_{t}$. When $M_{\bullet}>4 \times 10^{7} M_{\odot}$, the marginally bound orbit of Schwarzschild black hole becomes greater than the tidal radius, then the criterion for stellar depletion becomes $J<J_{\mathrm{mb}}$, where $J_{\mathrm{mb}}$ denotes the specific angular momentum for marginally bound geodesic. In general, $J_{\mathrm{mb}}$ is a function of black-hole spin and inclination relative to the equatorial plane, but for simplicity we adopt the orientation-averaged value $J_{\mathrm{mb}}=4 G M_{\bullet} / \mathrm{c}$ Kesden 2012) in the following calculation, where $c$ is the speed of light. For even greater black-hole mass $M_{\bullet} \gtrsim 10^{9} M_{\odot}$, tidal disruption occurs inside the event horizon of the central SMBH even when the black hole is maximally spinning, so no tidal flare could be produced by disrupting solar-type stars (Ivanov \& Chernyakova 2006; Kesden 2012).

As a result of tidal disruption and direct capture, a small fraction of stars are lost from the system during their pericenter passages. In a spherical system, the disruption rate of stars from distance $r$ to $r+d r$ from the central SMBH is approximately

$$
d \Gamma \simeq \frac{4 \pi r^{2} d r \rho(r)}{m_{*}} \frac{\theta^{2}(r)}{t_{d}(r)}
$$

(Frank \& Rees 1976; Syer \& Ulmer 1999), where $\rho(r)$ is the stellar mass density at $r, t_{d}(r)$ is the dynamical timescale, and $\theta^{2}(r)$ estimates the fraction of stars subjected to lose from the system. The loss fraction $\theta^{2}$ is dimensionless and can be interpreted geometrically as a solid angle, because at $r$ the lost stars have velocity vectors pointing toward the SMBH within an angle of $\theta_{\mathrm{lc}}(r)=J_{\mathrm{lc}} / J_{c}(r)$ and in an isotropic system their fraction is $\theta^{2}=\theta_{\mathrm{lc}}^{2}$. Here $J_{c}$ denotes the angular momentum for circular orbit and is of order $r \sigma(r)$ given the stellar velocity dispersion $\sigma(r)$. The cone-like region with half-opening angle $\theta_{\text {lc }}$ toward the $\mathrm{SMBH}$ is therefore called "loss cone". The isotropy of stellar distribution breaks down at the edge of loss cone when the orbital-averaged rms velocity deflection angle $\theta_{d}(r)$ is much smaller than $\theta_{\text {lc }}$ Lightman \& Shapiro 1977; Cohn \& Kulsrud 1978). Taking this effect into account, careful analysis of the loss-cone structure suggests that

$$
\theta^{2}=\min \left(\theta_{\mathrm{lc}}^{2}, \theta_{d}^{2} / \ln \theta_{\mathrm{lc}}^{-1}\right)
$$

Young 1977). Therefore, when $\theta_{d} \gg \theta_{\text {lc }}$ ("pinhole regime"), Equation (4) recovers $\theta^{2}=\theta_{\mathrm{lc}}^{2}$, because the stars act as if the loss cone does not exist and the system remains isotropic. On the other hand when $\theta_{d} \ll \theta_{\text {lc }}$ ("diffusive regime"), the loss cone becomes empty within one dynamical timescale, so afterwards only a fraction $\theta_{d}^{2} /\left|\ln \theta_{\mathrm{lc}}\right|$ of stars residing at the boundary layer $\theta_{\mathrm{lc}} \sim \theta_{\mathrm{lc}}+\theta_{d}$ of the loss cone will be depleted during one $t_{d}$. The total stellar disruption rate $\Gamma$ is an integration of Equation (3) over both pinhole and diffusive regimes.

To calculate $\rho(r), t_{d}(r)$, and $\theta^{2}(r)$, a physical model describing the stellar distribution in the host galaxy needs to be specified. We consider only the bulge component of a galaxy because it is 
the major source for stellar disruption. We model a bulge with a spherical model with double power laws, i.e.,

$$
\rho(r)= \begin{cases}\rho_{b}\left(r / r_{b}\right)^{-\gamma} & \left(r \leq r_{b}\right) \\ \rho_{b}\left(r / r_{b}\right)^{-\beta} & \left(r_{b}<r<r_{\max }\right) \\ 0 & \left(r \gtrsim r_{\max }\right)\end{cases}
$$

where $r_{b}$ is the break radius, $\rho_{b}$ is the stellar mass density at $r_{b}, \gamma$ and $\beta$ are, respectively, the inner and outer power-law indices, and $r_{\max }$ is the cut off radius to prevent divergence of the total stellar mass. The five model parameters, $\left(r_{b}, r_{\max }, \rho_{b}, \gamma, \beta\right)$, are determined by the following five physically motivated conditions

1. We define $r_{b}$ as the influence radius of $\mathrm{SMBH}^{1}$ such that the enclosed stellar mass is $2 M_{\bullet}$.

2. and 3. The values of $\gamma$ and $\beta$ are adopted from empirical galaxy models (Faber et al. 1997; Lauer et al. 2005) and will be specified explicitly in the following calculations. In our fiducial model, $\gamma=1.75$ and $\beta=2$, so that the galaxy has an inner Bahcall-Wolf and outer isothermal profile Bahcall \& Wolf 1976). By varying $\gamma$ and $\beta(\gamma, \beta<3)$, our simplified galaxy model could reconcile with a variety of real galaxies.

4. The total stellar mass enclosed in the radius $r_{\max }$ is $A M_{\bullet}$, where $A=400$ so that the SMBH-to-galaxy mass ratio satisfies the empirical correlation in the local university (e.g., Marconi \& Hunt 2003).

5. At the effective radius $r_{e}$, where the twodimensional (2-D) surface-density isophote encloses half of the total galaxy mass, the stellar velocity dispersion $\sigma_{e}$ satisfies the empirical correlation $M_{\bullet} \simeq 10^{8}\left(\sigma_{e} / 200 \mathrm{~km} \mathrm{~s}^{-1}\right)^{4} M_{\odot}$ (Tremaine et al. 2002). Note that the stellar mass enclosed by the 3-D sphere of radius $r_{e}$ is $M_{*}\left(r_{e}\right) \simeq(0.36,0.32) A M_{\bullet}$ when $\beta=(2,1.5)$, smaller than half of the galaxy mass.

\footnotetext{
${ }^{1}$ It is suggested that when $(\beta-\gamma) \gtrsim 1$, a practical definition for $r_{b}$ is that the mass deficit inside $r_{b}$ is $2 M_{\bullet}$ (Merritt 2006; Stone \& Loeb 2012), but the resulting $\rho_{b}$ differs from our fiducial value by only a factor of $(\beta-\gamma) /(3-\beta)$.
}

According to Jeans's equation in the isotropic limit

$$
\frac{d\left(\rho \sigma^{2}\right)}{d r}+\frac{G \rho\left[M_{*}(r)+M_{\bullet}\right]}{r^{2}}=0
$$

the velocity dispersion $\sigma \propto r^{-1 / 2}$ when $r \ll r_{b}$ and $\sigma \propto r^{1-\beta / 2}$ when $r \gg r_{b}$; therefore, we calculate $\sigma$ with

$$
\sigma(r)= \begin{cases}\sigma_{b}\left(r / r_{b}\right)^{-1 / 2} & \left(r \leq r_{b}\right) \\ \sigma_{b}\left(r / r_{b}\right)^{1-\beta / 2} & \left(r>r_{b}\right)\end{cases}
$$

where $\sigma_{b}$ is the velocity dispersion at $r_{b}$. By applying Equations (6) and (7) at $r_{e}$, we first derive

$$
r_{e}=\frac{A_{e}+1}{2 \beta-2} \frac{G M_{\bullet}}{\sigma_{e}^{2}}
$$

where $A_{e} \equiv M_{*}\left(r_{e}\right) / M_{\bullet}$ and $\sigma_{e}$ is computed with $M_{\bullet}-\sigma_{e}^{4}$ relation. Then the model parameters $\left(r_{b}, r_{\max }, \rho_{b}\right)$ are calibrated according to their definitions, and the results are $r_{b} \simeq r_{e}[(6-$ $\left.2 \gamma) /\left(3 A_{e}-\beta A_{e}\right)\right]^{1 /(3-\beta)}, r_{\max } \simeq\left(A / A_{e}\right)^{1 /(3-\beta)} r_{e}$, and $\rho_{b}=(3-\gamma) M_{\bullet} /\left(2 \pi r_{b}^{3}\right)$. For example, our fiducial galaxy model with $M_{\bullet}=10^{7} M_{\odot}, \gamma=1.75$, and $\beta=2$ corresponds to $r_{b} \simeq 4.5 \mathrm{pc}, r_{e} \simeq 260$ $\mathrm{pc}$, and $r_{\max } \simeq 820 \mathrm{pc}$.

Having specified the galaxy model, we now calculate the deflection angle $\theta_{d}$ which determines $\theta^{2}$ in Equation (44). Two-body scattering is an inherent relaxation mechanism in stellar system and it gives a lower limit of $\theta_{2}=J_{2} / J_{c}$ to $\theta_{d}$, where $J_{2}$ is the cumulative change of $J$ due to two-body scattering during one dynamical timescale. Because successive two-body scatterings are uncorrelated (incoherent), we have $J_{2}=\left(t_{d} / t_{r}\right)^{1 / 2} J_{c}$, where

$$
\begin{aligned}
t_{\mathrm{r}}(r)= & \frac{\sqrt{2} \sigma^{3}(r)}{\pi G^{2} m_{*} \rho(r) \ln \Lambda} \\
= & \frac{2 \sqrt{2} B^{2}}{(3-\gamma) \ln \Lambda} \frac{M_{\bullet}}{m_{*}} \times \\
& \left(\frac{\sigma}{\sigma_{b}}\right)^{3}\left(\frac{\rho}{\rho_{b}}\right)^{-1} \frac{r_{b}}{\sigma_{b}}
\end{aligned}
$$

is the two-body relaxation timescale, $\ln \Lambda$ is the Coulomb logarithm (we assumed a fiducial value of 5), and

$$
B \equiv \frac{r_{b}}{G M_{\bullet} / \sigma_{b}^{2}} \simeq \frac{3-\gamma}{(3-\beta)(\beta-1)}
$$

is a correction factor of order unity. When twobody scattering dominates the relaxation process, 
$J_{2}$ is an increasing function of $r$, with the transition between pinhole and diffusive regimes $\left(J_{2} \sim\right.$ $\left.J_{\text {lc }}\right)$ being situated at $r \sim r_{b}$. The differential loss rate $d \Gamma / d r$ (eq. (3) ) scales as $r^{9 / 2-2 \gamma}$ in the diffusive regime $\left(r \ll r_{b}\right)$ and as $r^{-1-\beta / 2}$ in the pinhole one $\left(r \gg r_{b}\right)$; therefore, the stellar disruption rate peaks at the transition regime at $r \sim r_{b}$.

Take our fiducial model with $M_{\bullet}=10^{7} M_{\odot}$, $\gamma=1.75$, and $\beta=2$ for example. The critical radius where $\theta_{2}^{2}=\theta_{\mathrm{lc}}^{2}$ is $r_{\text {cri }} \simeq 2.3 r_{b}$, and the total disruption rate due to two-body relaxation is $\Gamma \simeq 2.3 \times 10^{-5} \mathrm{yr}^{-1}$, consistent with previous calculations (e.g., Magorrian \& Tremaine 1999; Sver \& Ulmer 1999; Wang \& Merritt 2004; Brockamp et al. 2011). If $M_{\bullet}$ increases, $r_{\text {cri }} / r_{b}$ will also increase, given the fact that $\theta_{\mathrm{lc}}^{2}$ is a decreasing function of $r / r_{b}$, and that $\theta_{2}^{2} \propto M_{\bullet}^{-1}$ and $\theta_{\mathrm{lc}}^{2} \propto M_{\bullet}^{1 / 3}$ at any $r / r_{b}$. On the other hand, the integrated stellar-disruption rate will decrease, mainly because the diffusive regime of loss cone becomes larger. A more accurate calculation of $\Gamma$ could be carried out by solving the diffusion equation in the 2 -D $\mathcal{E}-J$ space (e.g., Lightman \& Shapiro 1977; Cohn \& Kulsrud 1978; Magorrian \& Tremaine 1999; Wang \& Merritt 2004), but it is considerably time-consuming and out of the scope of this paper. Nevertheless, the present scheme gives good approximation to the two-body disruption rate, and is sufficient to provide references for the sake of investigating the effects of galaxy mergers on the stellar-disruption rate.

\section{Enhanced loss-cone feeding during galaxy merger}

Because the loss cone is already "full" in the pinhole regime, enhancing relaxation efficiency in this regime does not increase the fraction of losscone stars, therefore would not increase stellardisruption rate. On the other hand, the loss cone in the diffusive regime is largely empty, so the disruption rate can be enhanced if stellar relaxation in this regime becomes more efficient. Enhancement of stellar relaxation in the diffusive regime can be achieved by galaxy merger due to at least two processes. First, perturbation by the companion galaxy would secularly change the stellar angular momenta (Roos 1981). Second, the triaxial gravitational potential built up during merger (Preto et al. 2011; Khan et al. 2011) would drive stars to galaxy center in a chaotic manner (Poon \& Merritt 2001). In this section we calculate the stellar-disruption rates due to the above two processes, and we show the rate for each of the two SMBHs in the merging system.

\subsection{Basic Theory}

A companion galaxy would tidally torque the stellar orbits in the central galaxy, secularly changing the orbital elements. Given mass $M_{p}$ of the perturber and its distance $d$ from central galaxy, one can derive $G M_{p} r / d^{3}$ for the tidal force exerted by $M_{p}$ across a stellar orbit of radius $r \ll d$ in the central galaxy. The corresponding tidal torque on the stellar orbit is of magnitude $T_{p} \sim G M_{p} r^{2} / d^{3}$. Because of the tidal torque, the angular momentum of star changes coherently, i.e., $\Delta J \propto t$, up to a timescale $t_{\omega}$, where $t_{\omega}$ is determined by the shorter one between the dynamical timescale of the perturber and the apsidal precession timescale of the stellar orbit (Binney \& Tremaine 2008). For $t>t_{\omega}$, the torque on stellar orbit adds up stochastically and in this case $\Delta J^{2} \propto t$. Therefore, averaged over a timescale much longer than $t_{\omega}$, the tidal torque changes $J^{2}$ by an amount of $J_{p}^{2}=$ $T_{p}^{2} t_{\omega} t_{d}(r)$ during each stellar dynamical timescale. As a result, the deflection angle $\theta_{d}^{2}$ in Equation (4) increases by an amount of $\theta_{p}^{2}=\left(J_{p} / J_{c}\right)^{2}$. We note that the calculation of $J_{p}$ is analogous to the calculation of angular-momentum change due to resonant relaxation where the resonance torque is induced by the grainy gravitational potential (Rauch \& Tremaine 1996; Hopman \& Alexander 2006).

Galaxy merger also increases the triaxiality of the gravitational potential (Preto et al. 2011; Khan et al. 2011). Poon \& Merritt (2001) showed that when the triaxiality is large, a consistent fraction of stars are fed to the loss cone in a chaotic manner and the loss cone remains full. Suppose $f_{c}$ is the fraction of stars on chaotic orbits, the extra contribution to stellar-disruption rate can be calculated by replacing $\theta_{d}^{2}$ with $\theta_{c}^{2}=f_{c} \theta_{\mathrm{lc}}^{2} \ln \theta_{\mathrm{lc}}$ (Merritt \& Poon 2004). It has been shown that $f_{c}$ approaches unity when the triaxiality becomes greater than 0.25 , but will rapidly decrease to 0 inside the influence radius of the central SMBH where the gravitational potential is largely spherical (Poon \& Merritt 2004). 
Because of tidal perturbation and triaxiality during galaxy merger, the effective deflection angle $\theta_{d}^{2}$ increases to

$$
\theta_{d}^{2}=\theta_{2}^{2}+\theta_{p}^{2}+\theta_{c}^{2}
$$

and in the diffusive regime the loss-cone-limited deflection angle ( $\theta^{2}$ in Equation (4) ) also becomes larger. Consequently, an enhancement of stellardisruption rate is anticipated. Now we have prepared Equations (3), (4), and (12) to calculate the stellar-disruption rate in merging galaxies. However, the equations are valid only in the adiabatic approximation, i.e., the gravitational potential varies on a timescale much longer than the typical timescale for stellar orbital evolution. If the adiabatic condition is violated, the galaxy core will be subject to significant heating and expansion on the dynamical timescale (Ostriker 1972; Merritt \& Cruz 2001; Bovlan-Kolchin \& Ma 2007). For the stars at $r \sim r_{b}$ which predominate the loss-rate enhancement, the maximum timescale of coherent angular-momentum change, $t_{\omega}$, is limited by the apsidal precession timescale, which is of order $t_{d}\left(r_{b}\right)$. The timescale for chaotic orbital evolution is also of order $t_{d}\left(r_{b}\right)$. The adiabatic limit therefore requires the orbital period of the merging galaxies to be longer than $t_{d}\left(r_{b}\right)$. For this reason, the following calculations are restricted to $d>2 r_{b}$.

\subsection{Stellar-disruption Rates}

We now calculate the stellar disruption rate for both galaxies in a merger. The black-hole and bulge components are modeled with the parameters $\left(M_{\bullet}, \gamma, \beta\right)$, as is described in $\S 2$ The mass ratio of the galaxies, by construction, equals the mass ratio of the SMBHs, $q \equiv M_{\bullet, s} / M_{\bullet, m} \leq 1$, where the subscript $m$ denotes the quantity for the bigger main galaxy and $s$ for the smaller satellite galaxy. As we have shown that the contribution to stellar tidal disruptions is dominated by the stars at the break radius of galaxy, we can approximately construct a merger system of galaxies without loss of generality as follows. Given the distance $d$ between the two galaxy centers, the total stellar density at any location is approximated by summing the densities of the two unperturbed bulges. In this density field, each galaxy approximately preserves its initial structure out to a radius $\min \left(r_{\max }, r_{\mathrm{tr}}\right)$, where $r_{\mathrm{tr}}$ is the truncation ra- dius due to mutual tidal interaction, defined by the condition that the mean densities within $r_{\mathrm{tr}}$ are the same for the two truncated galaxies. Figure 1 shows the density contours (upper panel), as well as the density distribution along the line connecting the two black holes (lower panel), for a merging system with $M_{\bullet}=10^{7} M_{\odot}, q=0.3$, and $d=50 r_{b}$.

Given the configuration of the merging system, we calculated $\theta_{2}$ and $\Gamma$ due to two-body relaxation for each of the two galaxies. To calculate $\theta_{p}$ and $\Gamma$ due to tidal perturbation, the perturber mass $M_{p}$ is derived by integrating the stellar and blackhole masses in the perturber galaxy enclosed by $r_{\mathrm{tr}}$. Note that the perturber is the satellite galaxy when calculating $\Gamma$ for the main galaxy, but can also be the main galaxy when calculating $\Gamma$ for the satellite. To calculate $\theta_{c}$ and $\Gamma$ due to chaotic loss-cone feeding, the triaxiality of galaxy needs to be determined. But our model is axisymmetric by construction, so the triaxiality cannot be derived self-consistently. We circumvent this inconsistency by assuming that at any radius where the density increment induced by the perturber excesses $\delta=20 \%$ of the initially unperturbed density, a fraction of $f_{c}=50 \%$ of stellar orbits are chaotic. Otherwise $f_{c}=0$ if $\delta<20 \%$. The radial range where $f_{c}=50 \%$ is insensitive to the choice of $\delta$ because of the steep density profiles we adopted in the following calculations.

Figure 2 shows the stellar disruption rates as a function of $d$ for both main (upper panel) and satellite (lower panel) galaxies. The parameters are $\left(M_{7}, q, \gamma, \beta\right)=(1,0.3,1.75,2)$ by default. When $d \gg 100 r_{b} \approx 450 \mathrm{pc}$, the loss-cone filling in both galaxies is dominated by two-body relaxation (dotted lines) and the disruption rate is identical to that for isolated single $\mathrm{SMBH}$. As the distance shrinks to $d \sim 100 r_{b}$, about $2 r_{e}$ of the central galaxy, the disruption rates induced by companion galaxies start to exceed those due to two-body relaxation. This is because $\theta_{p}\left(r_{b}\right)$ becomes greater than $\theta_{2}\left(r_{b}\right)$. As $d$ further decreases to $d \lesssim 10 r_{b} \approx 45 \mathrm{pc}, \theta_{c}\left(r_{b}\right)$ becomes greater than $\theta_{2}\left(r_{b}\right)$, so the contribution to $\Gamma$ due to triaxial potential starts to exceed that due to two-body relaxation. When the two galaxy cores are as close as the break radius of the main galaxy, $\Gamma$ in both galaxies have been enhanced by two orders of magnitude. In the subsequent evolution 


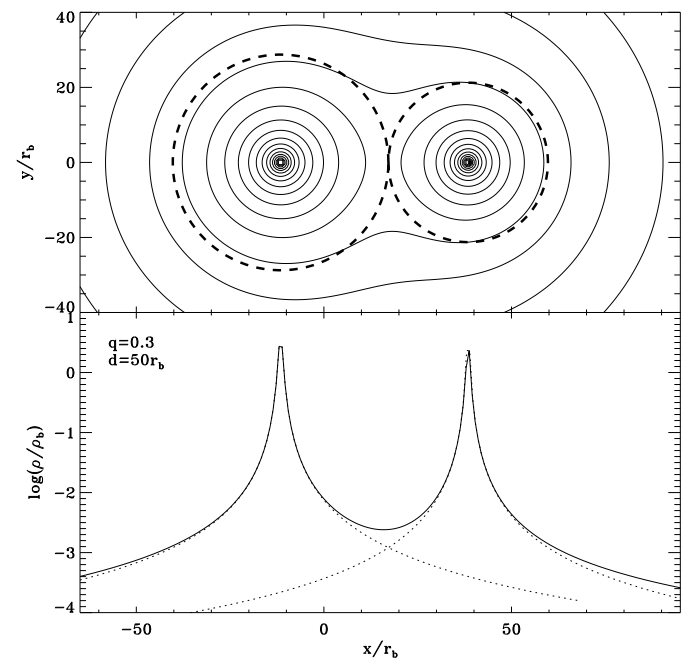

Fig. 1.- Upper: density contour in the mid-plane of a merging system with $M_{\bullet}=10^{7} M_{\odot}$ and $q=0.3$. The two galaxies, both have $\gamma=1.75$ and $\beta=2$, are separated by $50 r_{b}$ where $r_{b}$ refers to the break radius of the main (bigger) galaxy. The dashed circles mark the tidal truncation radii. Lower: density distribution along the line connecting the two black holes (solid curve). The dotted lines show to the initially unperturbed density distributions. with $d \lesssim 2 r_{b}$ for which our simple scheme cannot be applied, the three-body interactions between the two gravitationally bound SMBHs and the surrounding stars are expected to play an important role and to further enhance the disruption rates (Ivanov et al. 2005; Chen et al. 2009, 2011; Wegg \& Nate Bode 2011).

In a real merger, because galaxy orbitals are eccentric (Jiang et al. 2008), the distance $d$ will not decrease monotonically, but oscillate between the apocenter distance $r_{\text {apo }}$ and pericenter distance $r_{\text {per }}$, both distances decreasing with time due to dynamical friction. In this case, one can average the stellar-disruption rate over one orbital period according to $\bar{\Gamma}=\int_{r_{\mathrm{per}}}^{r_{\mathrm{apo}}} \Gamma(r) v_{r}^{-1} d r / \int_{r_{\mathrm{per}}}^{r_{\mathrm{apo}}} v_{r}^{-1} d r$, where $v_{r}$ denotes the radial velocity of galaxy at distance $r$. Since in our model, where $r / v_{r} \propto r^{\beta / 2}$ and $\Gamma(r) \propto r^{-\eta}$, both $\beta$ and $\eta$ are of order unity (see Section 3.3), we find that $\bar{\Gamma}$ differs from $\Gamma(d)$ by a factor of also order unity if we define $d \equiv\left(r_{\text {per }}+r_{\text {apo }}\right) / 2$. In this sense, the rates in Figure 2 can be used as the orbital-averaged stellardisruption rates for galaxy mergers with eccentric orbits. We also note that we may have underestimated the contribution from triaxial potential, because in our model by construction $f_{c}$ vanishes inside about the influence radius of black hole, as the stellar-density variation $\delta$ inside the sphere of radius $r_{b}$ is small (e.g. lower panel of Figure (1). In real galaxies, however, chaotic orbits may partially exist inside the influence radius of black hole (Poon \& Merritt 2001).

\subsection{Dependence of disruption rate on model parameters}

In $\S 3.2$, we have shown that tidal perturbation by the companion galaxy dominates the enhancement of $\Gamma$ in a merger. The enhancement occurs when $\theta_{p}$ in the diffusive regime exceeds $\theta_{2}$. As a result, the critical radius $r_{\text {cri, } p}$ that separates the pinhole and diffusive regimes is now determined by $\theta_{p}\left(r_{\mathrm{cri}, p}\right)=\theta_{\mathrm{lc}}$, and enhancement of stellar-disruption rate requires that $r_{\text {cri, } p}<r_{\text {cri }}$. Now we investigate in what mergers the condition $r_{\text {cri }, p}<r_{\text {cri }}$ would be satisfied. 
According to $J_{p}\left(r_{\text {cri,p }}\right)=J_{\text {lc }}$ and the relation

$$
\begin{gathered}
\frac{M_{\bullet}}{M_{p}} \propto q^{3\left(1-\beta_{p}\right) /\left(2 \beta_{p}\right)}\left(\frac{C_{p}}{C}\right)^{3\left(3-\beta_{p}\right) / \beta_{p}} \times \\
\left(\frac{d}{r_{b}}\right)^{\beta\left(\beta_{p}-3\right) / \beta_{p}},
\end{gathered}
$$

where

$$
C \equiv \frac{r_{b}}{G M_{\bullet} / \sigma_{e}^{2}} \simeq B\left[\frac{A_{e}(3-\beta)}{6-2 \gamma}\right]^{(2-\beta) /(3-\beta)},
$$

we first derive the following scaling relation in the limit $r_{\text {cri,p }} \lesssim r_{b}$ and $J_{\text {lc }}=J_{\text {td }}$ for the central galaxy:

$$
\begin{aligned}
& \frac{r_{\mathrm{cri}, \mathrm{p}}}{r_{b}} \propto B^{1 / 7} C^{-1 / 7} M_{\bullet}^{-1 / 42} q^{3\left(1-\beta_{p}\right) /\left(7 \beta_{p}\right)} \times \\
& \left(\frac{C_{p}}{C}\right)^{6\left(3-\beta_{p}\right) /\left(7 \beta_{p}\right)}\left(\frac{d}{r_{b}}\right)^{2 \beta\left(\beta_{p}-3\right) /\left(7 \beta_{p}\right)+6 / 7}
\end{aligned}
$$

Since $1<\beta<3$ for the majority of galaxies (Lauer et al. 2005), Equation (15) suggests that in general enhancement of $\Gamma$ would occur when the perturbing galaxy is larger or the galaxy distance is smaller. When tidal perturbation dominates the loss-cone filling, according to Equation (3) and $M_{\bullet} \propto \sigma_{e}^{4}$, the rate $\Gamma$ in the limit $r_{\text {cri,p }} \lesssim r_{b}$ scales as

$$
\Gamma \propto C^{-5 / 2} B^{-1 / 2} M_{\bullet}^{7 / 12}\left(r_{\text {cri, }} / r_{b}\right)^{1 / 2-\gamma} .
$$

For our fiducial model with $\gamma=7 / 4$ and $\beta=2$, we can derive $\Gamma \propto\left(d / r_{b}\right)^{-5 / 7}$, which is consistent with the numerical results given by the dashed lines at $d<30 r_{b}$ in Figure 2.

Figure 3 shows the dependence of $\Gamma$ on black hole mass when $q=0.3$. Equations (15) and (16) suggest that $\Gamma \propto M_{\bullet}^{(24+\gamma) / 42}$ when $q$ and $d / r_{b}$ are fixed. The enhanced stellar-disruption rates in Figure 3 generally agree with this scaling when $M_{\bullet} \lesssim 4 \times 10^{7} M_{\odot}$. When $M_{\bullet}>4 \times 10^{7} M_{\odot}$, the dependence of $\Gamma$ on $M_{\bullet}$ steepens because direct capture of stars by SMBH (GR effect) becomes important, such that the scaling of loss-cone size changes from $J_{\mathrm{lc}}^{2} \propto M_{\bullet}^{4 / 3}$ to $J_{\mathrm{lc}}^{2} \propto M_{\bullet}^{2}$. When $M_{\bullet} \gtrsim 10^{8} M_{\odot}$, the loss-cone stars will be directly captured by the central SMBH without producing tidal flares if the SMBH is non-rotating or rotates slowly (Ivanov \& Chernvakova 2006; Kesden 2012), and the corresponding curves are shown in

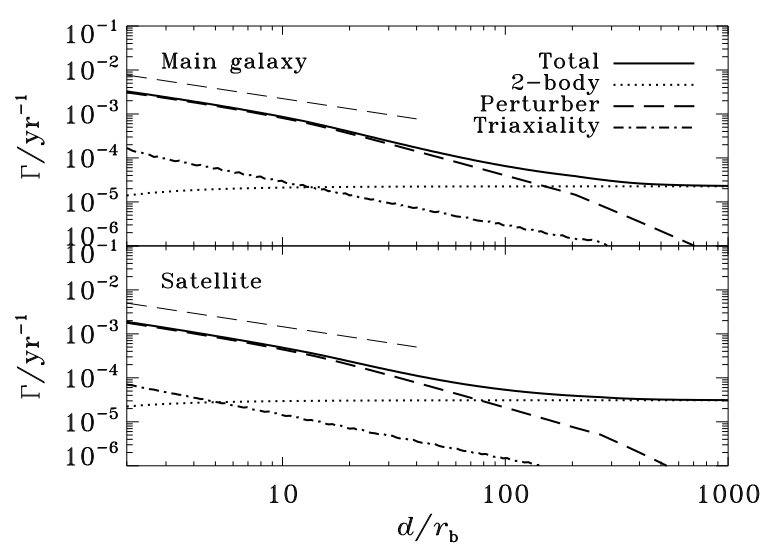

Fig. 2.- Stellar disruption rates as a function of galaxy separation for main (upper) and satellite (lower) galaxies. The dotted, dashed, and dashdotted lines refer to rates induced by, respectively, two-body relaxation, tidal perturbation, and triaxial gravitational potential. The thin dashed lines show the analytical solution $\Gamma \propto d^{-5 / 7}$ in arbitrary units derived in Section 3.3. The model parameters are $\left(M_{7}, q, \gamma, \beta\right)=(1,0.3,1.75,2)$ and $r_{b}$ refers to the break radius of the main galaxy.

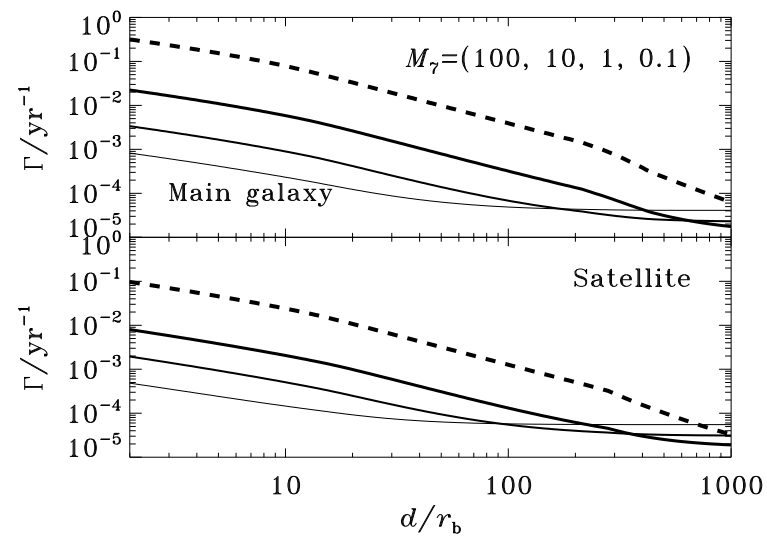

Fig. 3.- Total stellar disruption rates as a function of galaxy separation for different black hole masses. The model parameters are the same as in Figure 2, except that $M_{7}=(100,10,1,0.1)$ from top to bottom with decreasing line thickness. The dashed lines indicate that central SMBHs are more massive than $10^{8} M_{\odot}$ and stars fall into the SMBHs without tidal disruption. 
dashed lines. Note that even when the SMBH in the main galaxy is more massive than $10^{8} M_{\odot}$, the merging system could still produce tidal flares, due to the existence of a smaller SMBH in the satellite galaxy. We found that when $1<M_{7} \lesssim 10$, the event rates of tidal flares can be as high as $\sim 10^{-2} \mathrm{yr}^{-1}$ as $d$ shrinks to about $r_{b}$.

Figure 4 shows the dependence of $\Gamma$ on the mass ratio $q=M_{\bullet, s} / M_{\bullet, m} \leq 1$ of the two black holes, while $M_{\bullet, m}$ is fixed. For both main and satellite galaxies, the enhancement of $\Gamma$ becomes more significant as $q$ increases. It is worth noting that even $q$ is as small as 0.01 , the stellar disruption rate in the main galaxy can still be enhanced by two orders of magnitude when $d$ shrinks to about $r_{b}$. We also find that the enhanced stellar disruption rate in the satellite is more sensitive to $q$ than that in the main galaxy. This is because the baseline stellar-disruption rate, i.e., the rate for single black hole in isolated galaxy, changes with $q$ for satellite galaxy, but does not vary for the main galaxy since in the calculation $M_{\bullet}, m$ is fixed (e.g. see Equation 16). Quantitatively speaking, according to Equations (15) and (16), when varying $q$ while keeping $M_{\bullet, m}$ fixed, the enhanced stellar disruption rate for the main galaxy scales as $q^{3\left(1-\beta_{s}\right)(1-2 \gamma) /\left(14 \beta_{s}\right)}$, while the rate for the satellite scales as $q^{\left(24+\gamma_{s}\right) / 42-\left(1-2 \gamma_{s}\right)\left(3+\beta \beta_{s}-3 \beta_{s}\right) /(14 \beta)}$. For example, given $\left(\gamma, \beta, \gamma_{s}, \beta_{s}\right)=(1.75,2,1.75,2)$, one can derive $\Gamma \propto q^{15 / 56}$ for the main galaxy and $\Gamma \propto q^{59 / 84}$ for the satellite.

Figure 5 shows the variation of stellar disruption rate when the density profile of the main galaxy changes. For the main galaxy, when the inner power-law index $\gamma$ decreases from 1.75 to 1 , the stellar disruption rates due to two-body relaxation and tidal perturbation both drop by a factor of a few, because of the slight decrement of the stellar density at $r \sim r_{b}$. Meanwhile, the dependence of $\Gamma$ on $d / r_{b}$ at $d \lesssim 10 r_{b}$ changes from $\left(d / r_{b}\right)^{-5 / 7}$ to $\left(d / r_{b}\right)^{-2 / 7}$, resulting in an even smaller rate at $d \sim r_{b}$. When the outer power law index $\beta$ decreases from 2 to 1.5 , the stellar disruption rates in the main galaxies drop approximately by a factor of 20 . This is because the galaxy with shallower outer density profile is more spatially extended and has lower central density. For the satellite, when $\gamma$ or $\beta$ of the main galaxy decrease, the enhancement of stellar disruption rate occurs at smaller $d / r_{b}$ and becomes weaker for a

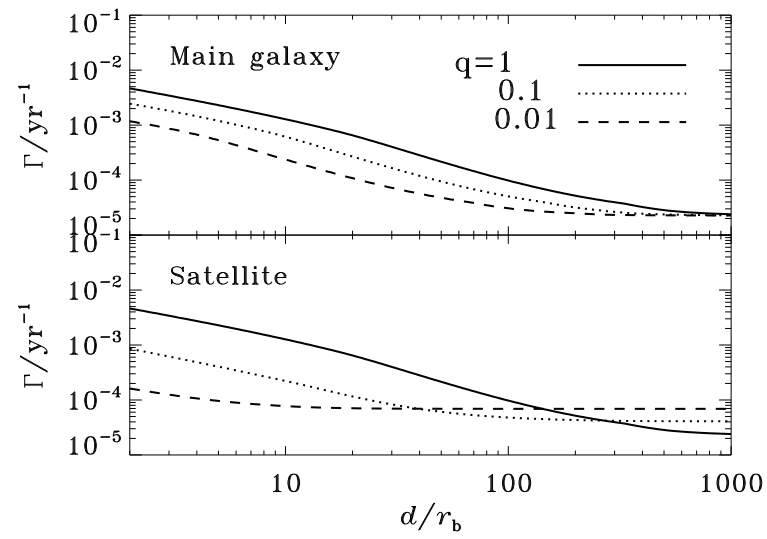

Fig. 4.- Stellar disruption rates as a function of galaxy separation for different $q$. The other parameters are the same as in Figure 2.

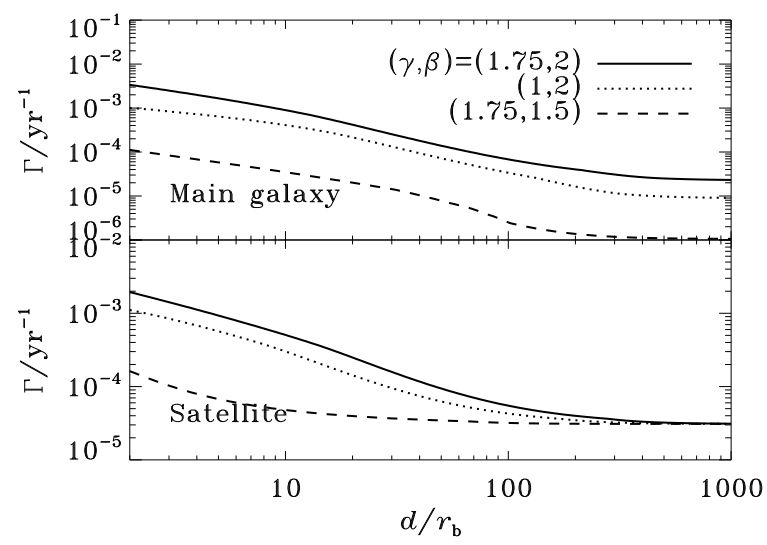

Fig. 5.- Stellar disruption rates as a function of galaxy separation for different density profiles in the main galaxy. The other parameters are the same as in Figure 2, 
fixed $d / r_{b}$. This is because $r_{b}$ of the main galaxy becomes greater as $\gamma$ or $\beta$ decrease, so that for the satellite the physical distance of the perturber increases if $d / r_{b}$ is fixed. We notice that when $\beta=1.5$, the stellar disruption rate in the main galaxy remains lower than that in the satellite as $d$ decrease. This result implies that in mergers where the main galaxies have low surface brightness, the tidal flares are mostly contributed by the satellite galaxies.

When the density profile of the satellite galaxy is varying, the resulting stellar disruption rates are shown in Figure 6. In general, the dependence of $\Gamma$ on the density profile can be understood in the light of the analysis for Figure 5. except that now the role between the main and satellite galaxies switches. However, one difference is that when $d$ shrinks to about $r_{b}$, the disruption rate in the main galaxy is not sensitive to the density profile of the satellite. This is because when $d \sim r_{b}$ the stellar cusp surrounding the $\mathrm{SMBH}$ in the satellite is almost completely striped off by the tidal filed of the main galaxy, so for the main galaxy the perturbing mass is approximately $M_{\bullet, s}$.

Figures 26 showed that galaxy merger starts enhancing stellar-disruption rate when the two galactic nuclei are still widely apart, well before the two SMBHs become gravitationally bound. The boost factor for each $\mathrm{SMBH}$ incorporated is about $10^{2}\left(M_{\bullet} / 10^{7} M_{\odot}\right)\left(d / r_{b}\right)^{\mu}(q / 0.3)^{\nu}$, where $M_{\bullet}$ is the mass of the subject black hole, $r_{b}$ refers to the break radius of the more massive galaxy, and $\mu$ and $\nu$ are indices depending on the density profiles of the two galaxies. Less massive black holes have smaller boost factors because prior to merger they already have higher stellar-disruption rates. The exact boost factor depends on the stellar-disruption rate prior to galaxy merger, which deserves some discussion. When calculating $\Gamma$ for isolated galaxies, we considered only two-body relaxation but not more efficient relaxation processes, such as resonant relaxation, perturbation by massive objects, or relaxation processes in triaxial gravitational potential (e.g. Rauch \& Tremaine 1996; Perets et al. 2007; Merritt \& Poon 2004). Resonant relaxation enhances stellar-disruption rate only mildly, less than a factor of a few in typical galaxies (Rauch \& Ingalls 1998). Massive perturbs, such as molecular clouds and stellar-mass black holes, if highly concentrate inside the influence radius of an $\mathrm{SMBH}$, in principle could enhance the stellar-disruption rate by orders of magnitude (Perets et al. 2007). But such galactic nuclei could only be transient, because large concentration of massive perturbs normally corresponds to short relaxation timescale. On the other hand, weak triaxiality seems intrinsic to galaxies, suggesting that chaotic loss-cone feeding may be important prior to galaxy mergers. If we use Equation (119) in Merritt \& Vasiliev (2011) 2 to estimate the stellar-disruption rate induced by triaxial potential inside the black-hole influence radius, meanwhile use formulae derived in Sections 2 and 3.1 with $f_{c}=0.1$ to calculate the rate due to chaotic orbits outside the black-hole influence radius, then the total disruption rates for isolated fiducial galaxies become $\Gamma \simeq(5.8,8.9,47) \times$ $10^{-5} \mathrm{yr}^{-1}$ when $M_{\bullet}=\left(10^{6}, 10^{7}, 10^{8}\right) M_{\odot}$. For comparison, the rates due to two-body relaxation only are $(4.0,2.3,1.4) \times 10^{-5} \mathrm{yr}^{-1}$. The difference is the greatest in the case of $M_{\bullet}=10^{8} M_{\odot}$, because the "gap" between $r_{\text {cri }}$ and $r_{b}$ is the largest. These results suggest that only in the most massive galaxies with $M_{\bullet} \gtrsim 10^{8} M_{\odot}$ could intrinsic triaxiality make the enhancement of stellardisruption rate less significant.

\section{Contributions of tidal flares by merging galaxies}

In a synoptic sky survey, the probability of catching tidal flares in merging galaxies does not depend only on the stellar-disruption rate, but also on the duration of galaxy mergers. In other words, the fractions of tidal flares in merging and in normal galaxies are proportional to the numbers of stellar-disruption events produced during, respectively, the merger and the quiescent phases. Since the duration of a galaxy merger is determined by the dynamical friction timescale, $t_{\mathrm{df}}$, the fraction of tidal flares in merging galaxies is proportional to the typical number of tidal stellar disruptions, $n=t_{\mathrm{df}} \Gamma$. Given the distance $d$ between two merging galaxies, we calculate the dynamical friction

2 Merritt \& Vasiliev (2011) used $\gamma=1.5$ to derive the stellardisruption rates. To derive rates for different $\gamma$, we used the scaling relation between $r_{\text {crit }}$ and $\gamma$ above Equation (116) in their paper. 
timescale as

$$
t_{\mathrm{df}}(d)=\left|\frac{d}{\dot{d}}\right| \simeq \frac{M_{g}}{M_{s}} \frac{t_{d}(d)}{\ln \left(M_{g} / M_{s}\right)},
$$

(see eq. [8.13] in Binney \& Tremaine 2008), where $M_{g}(d)$ here refers to the stellar mass enclosed by the radius $d$ in the main galaxy and $M_{s}$ is the total mass of the truncated satellite. When the two galaxies are distant and $\Gamma$ is not enhanced, the total number of disrupted stars is proportion to the dynamical friction timescale, which is

$$
t_{\mathrm{df}} \simeq q^{-1} t_{d}(d) / \ln \left(M_{g} / M_{s}\right)
$$

We refer to this early evolutionary stage as phase I. During phase I, the dependence of $t_{\mathrm{df}} \Gamma$ on $d^{\beta / 2}$ implies that the majority of tidal flares are contributed by wide galaxy pairs. When loss-cone feeding is enhanced due to tidal perturbation by the companion galaxy, the stellar-disruption rate $\Gamma$ increases with decreasing $d$. We refer to this later evolutionary stage as phase II, and we note that main and satellite galaxies enter phase II at different times. During phase II, the dependence of $t_{\mathrm{df}} \Gamma$ on $d$ flattens compared to that in phase I, implying a enhanced detection rate of tidal flares in close galaxy pairs.

Figure 7 gives the typical number of disrupted stars $\left(t_{\mathrm{df}} \Gamma\right)$ as a function of $d$ in our fiducial model. In the calculation, we did not consider the decrease of stellar density due to tidal disruption, because the total mass of disrupted stars is negligible with respect to the stellar mass in the initial condition. In general, when the merger is in phase I, $t_{\mathrm{df}} \Gamma$ scales as $d$, as predicted. During this phase, $t_{\mathrm{df}} \Gamma$ is not sensitive to the total mass of the system as long as $q$ is fixed, because massive systems where $\Gamma$ is larger have shorter $t_{\mathrm{df}}$. Note that before the main galaxy (solid curve) enters phase II, the satellite galaxy (dashed curve) contributes comparable number of, if not more, tidal flares. This is because when two-body relaxation predominates the loss-cone filling, smaller galaxies have smaller diffusive loss cones, therefore will have higher stellar-disruption rates, as is explained in the end of Section 2. When the galaxy mergers enter phase II, which is marked by the dots, the $t_{\mathrm{df}} \Gamma$ curve flattens, indicating an enhanced contribution of tidal flares by closer galaxy pairs. During this phase, the contribution of tidal flares from

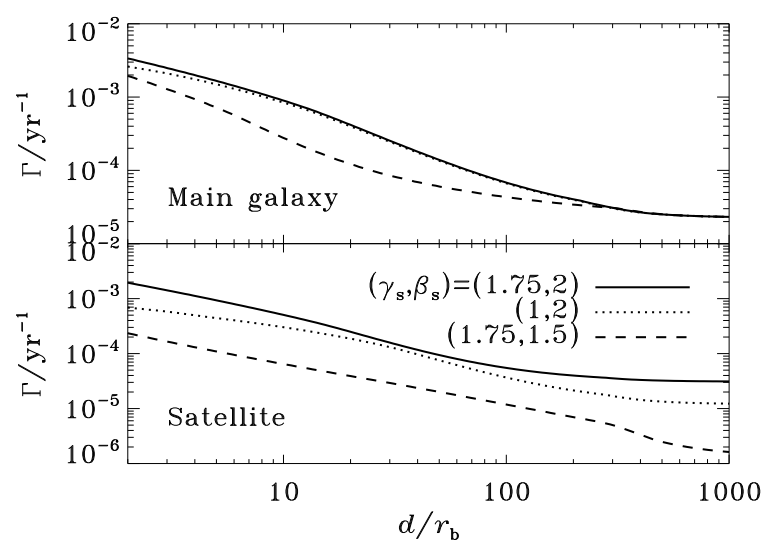

Fig. 6. - Same as Figure 5 but varying $\left(\gamma_{s}, \beta_{s}\right)$ in the satellite galaxy.

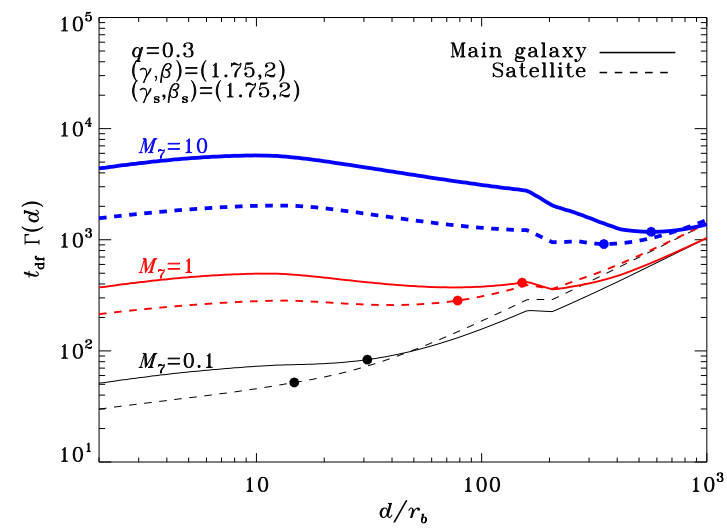

Fig. 7.- Typical number of disrupted stars contributed by main (solid) and satellite (dashed) galaxies at different separations. Lines with decreasing thickness refer to systems with decreasing $M_{7}$. The other parameters are the same as in Figure 2. 


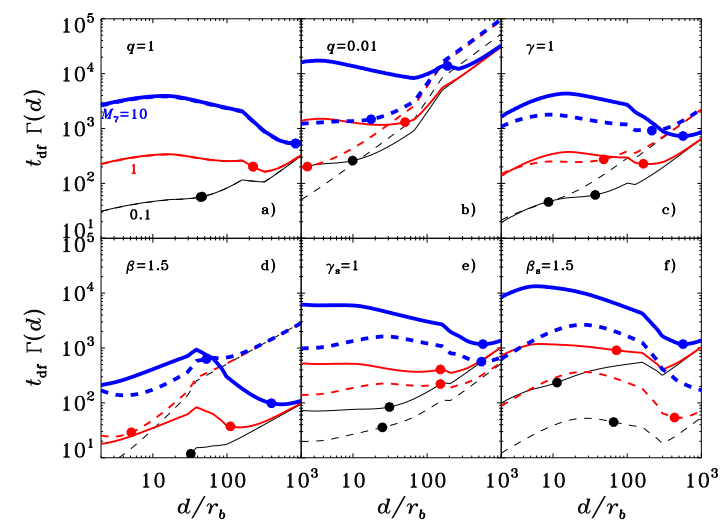

Fig. 8. - Same as Figure 7 but varying one model parameter, which is indicated at the upper-left corner of each panel.

main galaxy is typically greater than that from satellite.

Figure 8 shows the dependence of $t_{\mathrm{df}} \Gamma$ on different parameters of galaxy merger, which are summarized as follows.

1. Comparing panels (a) and (b), one can see that during phase $\mathrm{I}, t_{\mathrm{df}} \Gamma$ scales as $q^{-1}$, a characteristic relation due to dynamical friction. In phase II, as $q$ decreases from 1 to $0.01, t_{\mathrm{df}} \Gamma$ for the main galaxy increases by a factor of 10 relative to that in the fiducial case in Figure 7, but that for the satellite does not significantly change.

2. When $\gamma$ of the main galaxies decreases from 1.75 to 1 as shown in panel (c), in phase I the main galaxy contributes slightly less tidal flares compared to that in the fiducial model, because $\Gamma$ is smaller as $\gamma$ decreases. Meanwhile, the satellite contributes slightly more tidal flares because of longer $t_{\mathrm{df}}$. As a result, the relative contribution of tidal flares by the satellite becomes greater during phase I. During phase II, $t_{\mathrm{df}} \Gamma$ for both main and satellite galaxies increases as $d$ decreases from about $100 r_{b}$ to $10 r_{b}$. As $d$ becomes smaller than $10 r_{b}$, the typical number of stellar disruption decreases more steeply with smaller $d$ compared to that in the case with $\gamma=1.75$, because of less enhancement of stellar disruption rate as shown in Figure 5 .
3. Panel (d) shows that when $\beta$ of the main galaxy decreases from 2 to 1.5 , the number of tidal flares from the main galaxy drops during both phases I and II by about one order of magnitude relative to that in the fiducial case, because $\Gamma$ decreases significantly. While that from the satellite increases in phase I due to longer $t_{\mathrm{df}}$ and significantly drops during phase II because of greater physical distance between the galaxies. As a result, tidal flares are dominantly from the satellite galaxies during phase I, and almost equally contributed by the main and satellite galaxies during phase II.

4. Panel (e) indicates that when $\gamma_{s}$ of the satellite galaxy decreases from 1.75 to 1 , the tidal flares contributed by the satellite become slightly less than those in the fiducial case in both phases I and II. During phase II, when $2 r_{b} \lesssim d<10 r_{b}$, the number of tidal flares contributed by the main galaxy slightly increases relative to that in the fiducial case, because the satellite is more severely tidally truncated so that $t_{\mathrm{df}}$ becomes longer.

5 . When $\beta_{s}$ of the satellite galaxy decreases from 2 to 1.5 as shown in panel (f), the tidal flares contributed by the satellite during phase I are one order of magnitude less than those in the fiducial case. During phase II, the main galaxy contributes more tidal flares than in the fiducial case, because now 
the satellite is more susceptible to tidal stripping and $t_{\mathrm{df}}$ is much longer than that in the fiducial case.

Figures 7 and 8 suggest that during phase I the total number of disrupted stars scales roughly as

$$
n_{\mathrm{I}}(q, d) \sim 10^{3} q^{-1}\left(d / 10^{3} r_{b}\right)^{\beta / 2},
$$

insensitive to the total mass of the system or the stellar density profiles of the merging galaxies. During phase II, when the curve of $t_{\mathrm{df}} \Gamma$ is nearly independent of distance $d$, the total number becomes about

$$
n_{\mathrm{II}}\left(q, M_{7}\right) \sim 200 q^{m} M_{7}^{n},
$$

where $m \simeq 3\left(1-\beta_{s}\right)(1-2 \gamma) /\left(14 \beta_{s}\right)-1$ and $n=(45+\gamma) / 42$ are power-law indices derived from Equations (15), (16) and (18). It is worth noting that each merger investigated above involves only two galaxies. However, mergers of group galaxies are also common and the tidal disruption rates are expected to be even more heavily enhanced because of stronger perturbations and larger triaxiality.

\section{Discussions}

Formation of SMBHBs at galaxy centers is anticipated in the paradigm of hierarchical galaxy formation (Begelman et al. 1980), and coalescence of the binaries is predicted to induce recoiling velocities on the post-merger SMBHs (Centrella et al. 2010). In our previous works (Chen et al. 2008, 2009, 2011; Liu et al 2009), we investigated the possibility of using tidaldisruption flares to identify gravitationally bound SMBHBs of sub-pc separations in galactic nuclei. Recently, off-nuclear tidal flares have also been suggested in the literature to be probes of recoiling SMBHs (Komossa \& Merritt 2008; Merritt et al. 2009; Stone \& Loeb 2011, 2012; Li et al. 2012). However, an off-center tidal flare can also be produced by SMBHs embedded in merging galaxies. In this paper, we calculated the tidal flare rates produced by dual SMBHs in a particular evolutionary stage when the two SMBHs are still unbounded to each other and isolated in the cores of merging galaxies. We considered three major processes responsible for the loss-cone feeding in the merger system, namely, two-body stellar relaxation, tidal perturbation by the companion galaxy, and chaotic stellar orbits in triaxial gravitational potential.

By employing an analytical model to calculate the stellar disruption rates for both $\mathrm{SMBH}$ in the two merging galaxies, we found that prior to the formation of SMBHB the stellar disruption rate would be enhanced by as large as two orders of magnitude in both galaxies. The enhancement is dominated by tidal perturbation and occurs when the two galaxies are so close that the stars inside the influence radius of the central SMBH are significantly perturbed. We have shown that the enhanced stellar disruption rate depends on the masses, mass ratio, and density profiles of the two galaxies, as well as the distance $d$ between the two galaxy cores. In the fiducial model with $\left(M_{7}, q, \gamma, \beta\right)=(1,0.3,1.75,2)$, the enhancement starts when the perturber galaxy approaches approximately twice the effective radius of the central galaxy $\left(d \simeq 2 r_{e}\right)$. In more massive systems with $M_{7}>10$, where the stellar disruption rates due to two-body relaxation are generally lower, the enhancement starts as soon as $d$ shrinks to $10 r_{e}$. As a result, the phase with enhanced stellar-disruption rate extends to an evolutionary stage much earlier than the formation of bound SMBHB, which considerably increases the detection rate of wide SMBH pairs in tidalflare surveys. When $d$ shrinks to about the influence radius of the central SMBH $\left(d \sim 2 r_{b}\right)$, the stellar disruption rate in the fiducial model increases to $3 \times 10^{-3} \mathrm{yr}^{-1}$ in the main galaxy and to $2 \times 10^{-3} \mathrm{yr}^{-1}$ in the satellite. Compared to the peak rates in the later evolutionary stages with gravitationally-bound binary SMBHs (e.g. Chen et al. 2009, 2011; Wegg \& Nate Bode 2011), the total stellar-disruption rates before SMBHs become bounded are smaller by only a factor of a few. In more massive or equal-mass $(q>0.3)$ mergers, the stellar disruption rates could be even higher.

The above results showed that the tidal disruption rates by off-center SMBH pairs in merging galaxies are several order magnitudes higher than those by recoiling off-nuclear SMBHs (Komossa \& Merritt 2008; Li et al. 2012; (Stone \& Loeb 2012), implying that off-center tidal disruption flares would be overwhelmed by the SMBH pairs in merging 
galaxies. Therefore, it would be challenging to distinguish recoiling SMBHs in off-center tidal disruption flares. One possible way to distinguish the two kinds of off-center tidal disruptions may be to identify the evolutionary stages of galaxies. Recoiling SMBHs are in galaxies at late stages of mergers, while un-bounded SMBH pairs are in galaxies at early or middle stages of mergers. Early stages of major mergers when galaxies are widely separated may be identified with the disturbed morphology of host galaxies. However, morphological signatures of galaxy merger are weak during the middle or late stages of major mergers, as well as during the whole stages of minor mergers, therefore it would be also a challenge to identify these merger stages. Another difference may be among the properties of star clusters around the off-center SMBHs. A recoiling $\mathrm{SMBH}$ is expected to reside in an ultra-compact bound star cluster of mass much smaller than the black-hole mass, of size much smaller than the black-hole influence radius, and of stellar-velocity dispersion much larger than that of host galaxy (Merritt et al. 2009; Li et al. 2012). It may also associate with a massive cloud of unbound stars, whose mass is comparable to the black-hole mass, size comparable to the black-hole influence radius, and stellar-velocity dispersion comparable to or greater than that of the host galactic nuclei (Li et al. 2012). While the star clusters hosting the the secondary black holes in minor mergers are the remnants of the tidally truncated satellite galaxies. These clusters are orders of magnitude heavier than the secondary SMBHs, their sizes are much larger than the influence radii of the secondary or the primary SMBHs, and their stellarvelocity dispersions are comparable to those of typical dwarf galaxies but significantly smaller than those of the primary galactic nuclei. Therefore, the two types of star clusters should differ significantly in their sizes, stellar-velocity dispersions, and the mass ratios between SMBHs and star clusters, which could be identified with deep photometrical and spectroscopical observations.

When a pair of SMBHs evolve to about the influence radius, $d \sim r_{\mathrm{b}}$, the enhanced stellardisruption rates can be as high as $10^{-2} \mathrm{yr}^{-1}$. For such a high tidal disruption rate, multiple tidal flares may occur in the same galaxy within a time span of decades. Unlike the recurring tidal flares in binary or recoiling SMBH systems, the flares in merging galaxies are contributed by wide $\mathrm{SMBH}$ pairs separated by $r_{b} \sim 1-10 \mathrm{pc}$ (depending on black hole mass and galaxy density profile). Note that a separation of $10 \mathrm{pc}$ at redshift $z=0.1$ (1) corresponds to an angular size of 5 (1) milliarcsec (mas). As a result, spatial offsets between successive tidal flares in such a merging system may be detected by instruments such as Gaia and $L S S T_{3}^{3}$. Figures 3 and 4 imply that such flipflop flares could occur in the galaxy mergers with $10^{7} M_{\odot}<M_{\bullet}<10^{8} M_{\odot}$ and $q>0.1$. The mergers with $M_{\bullet}<10^{7}$ could not produce recurring flares because the stellar-disruption rate is too low. When $10^{7} M_{\odot}<M_{\bullet}<10^{8} M_{\odot}$ but $q<0.1$, most flares are produced in the main galaxy; therefore, the recurring flares are unlikely to display spatial offset, and would be indistinguishable from those in binary or newly-formed recoiling $\mathrm{SMBH}$ systems. When $M_{\bullet}>10^{8} M_{\odot}$, the $\mathrm{SMBH}$ in the main galaxy would directly capture stars, mostly without producing flares, while the $\mathrm{SMBH}$ in the satellite could still produce tidal flares if $M_{\bullet, s}<10^{8} M_{\odot}$. In the last case, although the recurring flares occur at the same sky position, they should be displaced from the center of the minor merger by an amount of $r_{b} \gtrsim 10$ pc. Sources with such high flaring rate and large off-center displacement cannot be produced by binary or recoiling SMBHs. The above discussions suggest that with the aid of telescopes with high spatial resolution, the cause of the recurring tidal flares can be distinguished.

In the universe, the fraction of tidal flares contributed by galaxy mergers is proportional to the total number of the disrupted stars during merger. During each merger, the number of tidal flares contributed by phase I, when the separations of galaxies are about $r_{e} \lesssim d \lesssim 10 r_{e}$, is about $n_{\mathrm{I}} \sim 10^{3} q^{-1}\left(d / 10^{3} r_{b}\right)^{\beta / 2}$ [Figures (7) and (8)]. The scaling $n_{\mathrm{I}} \propto q^{-1}$ implies that $n_{\mathrm{I}}$ is determined mainly by minor mergers. Suppose a galaxy experiences $N$ mergers during a Hubble time $\left(\sim 10^{10}\right.$ yr), then during one duty cycle, the number of tidal flares contributed by the isolation phase is about $n_{s} \sim 2 \times 10^{5} / N$, if two-body scattering is the dominant relaxation process. Since a galaxy

${ }^{3}$ See Section 3.7 of LSST Science Book Version 2.0,
http://www.lsst.org/lsst/scibook 
with $M_{7}=1$ (10) at redshift $z=0$ has experienced typically $N \sim 10$ (100) mergers and most mergers have $q \sim 0.1$ (Hopkins et al. 2010), according to the ratio $n_{\mathrm{I}}: n_{s}$, we find that about $\sim 5 \%(50 \%)$ tidal flares are contributed by phaseI galaxy mergers $\left(d \sim 10^{3} r_{b}\right)$.

For typical mergers with $q \ll 1$, according to Figure 8 , the majority of the tidal flares are produced in satellite galaxies during phase I, unless the satellite galaxies have low surface brightness. This result implies that a large fraction of genuine tidal flares would be displaced by several $r_{e}$ from the centers of the merging systems. Given that an offset of $2 r_{e} \sim 500 \mathrm{pc}$ corresponds to 250 (60) mas at $z=0.1$ (1), these offset tidal flares could be misidentified as supernovae or gammaray bursts by careless classification schemes. They may also be mistaken as "naked" recoiling quasars (e.g., Komossa \& Merritt 2008) or "orphan transients" (X-/ $\gamma$-ray transients either uncorrelated with bursts in low-energy bands or without detection of optical counterparts, e.g., Horan et al. 2009) because of the relative dimness of the satellites. The mis-identification could be very common in massive galaxies, because the physical scale of $r_{e}$ is larger.

During phase II when the stellar disruption rates are enhanced by galaxy mergers, the main galaxies would contribution typically more than half of the tidal flares, unless $q \sim 1$ or the surface brightnesses of the main galaxies are low. This result indicates that in an advanced merger, where the separation between the two galaxy cores is less than the effective radius of the main galaxy, the tidal flares preferentially reside in the massive nucleus of the system. According to Figures 7 and 8 the number of tidal flares contributed by such advanced merger phase does not depend on $d / r_{b}$, and scales as $n_{\mathrm{II}} \sim 200 q^{m} M_{7}^{n}$, where $m<0$ and $n>0$ are analytical indices derived in $\S$ 团 Therefore, the biggest contribution is expected to come from minor mergers in massive systems. Because $n_{\mathrm{II}}$ is typically less than $n_{\mathrm{I}}$, the contribution of tidal flares from phase II is typically smaller than that from phase I. However, for the most massive systems with $M_{7} \gtrsim 10$ in which the main SMBHs mostly swallow the stars without producing tidal flares, one major merger $(q>0.3)$, or one minor merger $(q \lesssim 0.3)$ between galaxies of low surface brightness, would produce more tidal flares in phase II than in phase I. In these particular systems, a greater fraction of tidal flares would be contributed by close SMBH pairs with separations $10 \lesssim d / r_{b} \lesssim 100$.

It is important to know the relative contributions of tidal flares by single $\left(n_{s}\right)$, binary $\left(n_{b}\right)$, and merging SMBH systems $\left(n_{\mathrm{I}}\right.$ and $\left.n_{\mathrm{II}}\right)$. The total number of flares produced by a recoiling black holes is typically smaller than $10^{3}$ (Komossa \& Merritt 2008; Stone \& Loeb 2011; Li et al. 2012), therefore negligible in the comparison. According to Chen et al. (2011), during the lifetime of an SMBHB with $q \ll 1$, the interaction between the binary and the surrounding dense stellar cusp will produce a number of $n_{b} \simeq 7 \times 10^{4} q^{(2-\gamma) /(6-2 \gamma)} M_{7}^{11 / 12}$ of tidal flares. Suppose a galaxy on average experiences $N$ mergers and $M(M \leq N)$ of them result in the formation of SMBHBs. Then being averaged by one duty cycle of galaxy merger, $n_{s}: n_{b}:\left(n_{\mathrm{I}}+n_{\mathrm{II}}\right)$ is about $20: 5 M: N$, where we used $q=0.1$ because minor mergers are the most common (Volonteri et al. 2003; Hopkins et al. 2010; McWilliams et al. 2013b). For galaxies of total masses $\left(10^{9}, 10^{10}, 10^{11}\right) M_{\odot}$, typical $N$ are $\left(1,10,10^{2}\right)$ (Hopkins et al. 2010) or significantly higher (McWilliams et al. 2013a, b; Bédorf \& Portegies Zwart 2013), while $M$ are predicted to be greater than 1 Volonteri et al. 2003). These numbers highlight the significant contribution of tidal flares from merging systems with multiple SMBHs. To give more accurate calculations, one has to combine the cosmic merger history of galaxies, as well as the formation rate of SMBHBs of different masses and mass ratios. Such calculations and the assessment of their uncertainties are beyond the scope of the current paper and will be addressed in a future paper.

We are grateful to Shuo Li, Zuhui Fan, Rainer Spurzem, and Thijs Kouwenhoven for helpful comments. We also thank Alberto Sesana for earlier discussions on this topic. This work is supported by the National Natural Science Foundation of China (NSFC11073002). F.K.L. also thanks the support from the Research Fund for the Doctoral Program of Higher Education (RFDP), and X.C. acknowledges the support from China Postdoc Science Foundation (2011M500001). 


\section{REFERENCES}

Bahcall J. N., \& Wolf R. A., 1976, ApJ, 209, 214

Bédorf, J., Portegies Zwart, S. 2013, MNRAS, in press (arXiv:1301.6784)

Begelman, M. C., Blandford, R. D., \& Rees, M. J., 1980, Nature, 287, 307

Binney, J., \& Tremaine, S. 2008, Galactic Dynamics: Second Edition, Princeton University Press, Princeton, NJ USA

Bloom, J. S., Giannios, D., Metzger, B. D., et al. 2011, Science, 333, 203

Boylan-Kolchin, M., \& Ma, C.-P. 2007, MNRAS, 374,1227

Brockamp, M., Baumgardt, H., \& Kroupa, P. 2011, MNRAS, 418, 1308

Burrows, D. N., Kennea, J. A., Ghisellini, G., et al. 2011, Nature, 476, 421

Cenko, S. B., Krimm, H. A., Horesh, A., et al. 2012, ApJ, 753, 77

Centrella, J., Baker, J. G., Kelly, B. J., \& van Meter, J. R. 2010, Rev. Mod. Phys., 82, 3069

Chen, X., Liu, F. K., \& Magorrian, J., 2008, ApJ, 676,54

Chen, X., Madau, P., Sesana, A., \& Liu, F. K. 2009, ApJ, 697, L149

Chen, X., Sesana, A., Madau, P., \& Liu, F. K. 2011, ApJ, 729, 13

Cohn, H., \& Kulsrud, R. M. 1978, ApJ, 226, 1087

Colpi, M. \& Dotti, M., 2011, Advanced Science Letters, 4, 181

Donley, J. L., Brandt, W. N., Eracleous, M., \& Boller, T. 2002, AJ, 124, 1308

Faber, S. M., Tremaine, S., Ajhar, E. A., et al. 1997, AJ, 114, 1771

Frank, J., \& Rees, M. J. 1976, MNRAS, 176, 633

Hills, J. 1975, ApJ, 254, 295

Hopkins, P. F., et al. 2010, ApJ, 724, 915
Hopman, C., \& Alexander, T., 2006, ApJ, 645,1152

Horan, D., Acciari, V. A., Bradbury, S. M., et al. 2009, ApJ, 695, 596

Hughes, S. A. 2009, ARA\&A, 47, 107

Gezari, S., Strubbe, L., Bloom, J. S., et al., 2009, Astro2010: The Astronomy and Astrophysics Decadal Survey, Science White Papers, no.88

Ivanov, P. B . \& Chernyakova, M. A., 2006, A\&A, 448,843

Ivanov, P. B., Polnarev, A. G., \& Saha, P., 2005, MNRAS, 358, 1361

Jiang, C. Y., Jing, Y. P., Faltenbacher, A., Lin, W. P., \& Li, C. 2008, ApJ, 675, 1095

Kesden, M. 2012, Phys. Rev. D, 85, 024037

Khan, F., Just, A., \& Merritt, D. 2011, ApJ, 732, 89

Komossa, S., 2002, Rev. Mod. Astron., 15, 27

Komossa, S., \& Merritt, D., 2008, ApJ, 683, 21

Lauer, T. R., et al. 2005, AJ, 129, 2138

Li, S., Liu, F. K., Berczik, P., Chen, X., \& Spurzem, R. 2012, ApJ, 748, 65

Lightman, A. P. \& Shapiro, S. L., 1977, ApJ, 211, 244

Liu, F. K., Li, S., \& Chen, X., 2009, ApJ, 706, L133

Magorrian, J. \& Tremaine, S., 1999, MNRAS, 309, 447

Marconi, A., \& Hunt, L., 2003, ApJ, 589, 21

McWilliams, S.T., Ostriker, J.P., \& Pretorius, F. 2013a, Science, submitted (arXiv:1211.4590)

McWilliams, S.T., Ostriker, J.P., \& Pretorius, F. 2013b, ApJ, submitted (arXiv:1211.5377)

Merritt, D. 2006, ApJ, 648, 976

Merritt, D., \& Cruz, F. 2001, ApJ, 551, L41

Merritt, D. \& Milosavljević, M., 2005, Liv. Rev. Rel., 8, 8 
Merritt, D. \& Poon, M. Y., 2004, ApJ, 606, 788

Merritt, D., Schnittman, J. D., \& Komossa, S. 2009, ApJ, 699,1690

Merritt, D., \& Vasiliev, E. 2011, ApJ, 726, 61

Ostriker, J. P., Spitzer, L., \& Chavalier, R. A., 1972, ApJ, 176, L47

Perets, H. B., Hopman, C., \& Alexander, T., 2007, ApJ, 656, 709

Poon, M. Y., \& Merritt, D., 2001, ApJ, 549, 192

Poon, M. Y., \& Merritt, D., 2004, ApJ, 606, 774

Preto, M., Berentzen, I., Berczik, P., \& Spurzem, R. 2011, ApJ, 732, L26

Rauch, K. P., \& Ingalls, B., 1998, MNRAS, 299, 1231

Rauch, K. P., \& Tremaine, S. 1996, NewA, 1, 149

Rees, M. J. 1988, Nature, 333, 523

Roos, N., 1981, A\&A, 104, 218

Schnittman, J. D. 2010, ApJ, 724, 39

Seto, N., \& Muto, T. 2011, MNRAS, 415, 3824

Stone, N., \& Loeb, A. 2011, MNRAS, 412, 75

Stone, N., \& Loeb, A. 2012, MNRAS, 422, 1933

Syer, D. \& Ulmer, A., 1999, MNRAS, 306, 35

Tremaine, S., et al. 2002, ApJ, 574, 740

van Velzen, S., Farrar, G. R., Gezari, S., et al. 2011, ApJ, 741, 73

Volonteri, M., Haardt, F., \& Madau, P. 2003, ApJ, 582,559

Wang, J.-X. \& Merritt, D., 2004, ApJ, 600, 149

Wegg, C., \& Nate Bode, J. 2011, ApJ, 738, L8

Young, P. J. 1977, ApJ, 215, 36

This 2-column preprint was prepared with the AAS LATEX macros v 5.2 . 